\title{
Importance of intensity-to-phase coupling for water-window high-order-harmonic generation with few-cycle pulses
}

\author{
Stephan M. Teichmann, ${ }^{1}$ Francisco Silva, ${ }^{1}$ Seth L. Cousin, ${ }^{1}$ and Jens Biegert ${ }^{1,2, *}$ \\ ${ }^{1}$ ICFO-Institut de Ciències Fotòniques, Mediterranean Technology Park, 08860 Castelldefels (Barcelona), Spain \\ ${ }^{2}$ ICREA-Institució Catalana de Recerca i Estudis Avançats, 08010 Barcelona, Spain
}

(Received 16 April 2015; published 16 June 2015)

\begin{abstract}
We present half-cycle cutoff features in the water window using high-order-harmonic generation (HHG) with two-cycle, carrier-envelope-phase-controlled pulses at $1.85 \mu \mathrm{m}$ in a high-pressure neon target. A relative carrier-envelope-phase (CEP) shift of the acquired spectra at different pressures is related to an in situ slip of the CEP in the HHG target. A simple model based on the pressure-dependent dispersion of the target gas reveals the importance of the free electrons for this effect. Our analysis also reveals that the effect is relaxed at high target pressures due to plasma defocusing of the driving pulse. We show that as a consequence of the CEP slip mediated by free electrons, moderate-intensity fluctuations can have a detrimental influence on the generation and application of attosecond pulses. Our findings emphasize the potential of combining high target pressures and long driver wavelengths to reveal subtle details of the complex light-matter interaction of HHG.
\end{abstract}

DOI: 10.1103/PhysRevA.91.063817

PACS number(s): 42.65.Ky, 33.20.Xx, 32.80.Wr

\section{INTRODUCTION}

High-order-harmonic generation (HHG) is a highly nonlinear light-matter interaction that has been studied extensively during the past two decades [1,2]. It offers the possibility to generate highly coherent short-wavelength radiation [extreme ultraviolet (XUV) and $\mathrm{x}$ rays] that has found application in high-resolution table-top imaging experiments [3], for example. In addition, the duration of the emitted bursts of radiation is a fraction of a cycle of the generating laser pulse, i.e., between tens and hundreds of attoseconds. Since the generation process is repeated in every half cycle with a sufficiently large field amplitude, trains of attosecond pulses are readily generated with HHG [4]. They have found use in measuring the time delay between photoionization from different shells of the argon atom [5], for example. For typical pump-probe experiments, the isolation of one of the attosecond pulses from the train is of interest. Several techniques have been introduced to achieve this [6-10], and they all have in common a gating mechanism, either spectral-temporal or spatial, to limit the emission to an individual burst. Such isolated attosecond pulses have formed the principle tool for the investigation of ultrafast dynamics in atomic [11-13], molecular [14-16], and solid-state [17-19] systems. Beyond this, HHG has been exploited for the investigation of the light-matter interaction itself, e.g., through observation of nonlinear pulse compression inside the HHG target gas [20].

Recently, substantial theoretical [21-24] and experimental [10,25-29] effort has gone into the efficient extension of the generated photon energies into the soft $\mathrm{x}$-ray spectral region. In particular the water window between the carbon and oxygen $K$ edges at 284 and $543 \mathrm{eV}$, respectively, presents an interesting bandwidth for spectroscopic applications due to the high absorption contrast between biological samples and their natural, aqueous environment [30]. It has been established that the efficient generation of water-window radiation, i.e., an appreciable photon flux that allows for applications with reasonable integration times, requires the

\footnotetext{
*jens.biegert@icfo.eu
}

combination of longer wavelengths than the $800 \mathrm{~nm}$ provided by the mature Ti:sapphire technology and multiatmosphere target pressures [27-29,31]. Following this approach of longwavelength-driven, high-pressure $\mathrm{HHG}$, photon energies up to $1.6 \mathrm{keV}$, or high-order harmonics up to order $\sim 5000$, could be detected [31].

Here, we report on the generation of water-window highorder harmonics employing few-cycle laser pulses with a center wavelength of $1.85 \mu \mathrm{m}$ and a high-pressure neongas target. By finely controlling the carrier-envelope phase (CEP) of the driving pulses and acquiring high-order-harmonic spectra as a function thereof at different target pressures, we can observe how the interaction medium leads to an in situ CEP shift. The magnitude of the shift is directly influenced by the high gas pressures as well as the long driver wavelength, presenting a new regime for the investigation of details of the light-matter interaction through the detection of high-order-harmonic spectra.

\section{BACKGROUND}

At the level of an individual atom or molecule, HHG may be described well with a semiclassical three-step model of the light-matter interaction [32,33]. Following this, the highest generated photon energy, the so-called cutoff, scales linearly with the intensity and quadratically with the wavelength of the driving pulse. While wavelength scaling of HHG to higher photon energies appears favorable based on the $\lambda^{2}$ law, quantum-mechanical calculations reveal an efficiency drop of the generated radiation that scales as $\lambda^{-(7-9)}$ [21-24]. In order to achieve a useful photon flux at high photon energies with long-wavelength-driven high-order harmonics, the macroscopic aspects of the interaction need to be exploited. Using high target-gas pressures, phase matching between the driving field and the source of the high-order harmonics may be achieved $[27,28]$ such that the reduced output of the individual emitters can be mitigated.

\section{A. Half-cycle cutoffs}

When few-cycle pulses are employed for HHG, two fundamental aspects of the three-step process are strongly 
affected, a decisive parameter being the CEP $\Phi$ of the pulse. First, the tunnel ionization rate varies drastically within the driving pulse, resulting in different ion fractions for subsequent half cycles. Since the amplitude of the emitted harmonics is in part determined by this ion fraction [34], the signal strength of different half cycles in a few-cycle pulse varies significantly. Second, the difference in electric field strength of subsequent half cycles translates into different return and therefore photon energies. Since the return energy scales with the square of the wavelength of the driving field, the half-cycle contrast of return energies is more striking at longer wavelengths. Therefore, the concept of half-cycle cutoffs (HCOs) becomes meaningful in the case of HHG with few-cycle pulses, denoting the highest photon energies emitted by different half-cycles.

The primary use of HCOs is the spectral optimization for the generation of isolated attosecond pulses [35-37]. If the spectrum between the two highest HCOs can be separated from the rest, i.e., spectrally filtered, only one half cycle contributes to the overall emission, resulting in an isolated attosecond pulse. HCO structures have also been exploited as a means to determine the wave form of the driving field on target [38-40]. In these experiments, simulated high-order-harmonic spectra were fitted to measured HCO traces, using the CEP, the chirp, and the peak intensity as fit parameters. Recently, HCOs were also reported for the first time in the water window at $300 \mathrm{eV}$ [41] and at $400 \mathrm{eV}$ [29].

In the present work, we use the strong dependence of HCOs on the electric-field wave form to observe the effect of the generation medium on the CEP.

\section{B. In situ CEP slip}

When a pulse travels through material, dispersion leads to a CEP slip due to the difference between the phase and group velocities [42]. Thus, at sufficiently high gas-target pressures, we can expect that the HHG interaction medium influences the CEP of a few-cycle driving pulse. If the gas target is of length $L$ and the driving pulse of wavelength $\lambda$ travels in the $z$ direction, the dispersion-induced phase slip is given by [2]

$$
\begin{aligned}
\Delta \Phi & =\frac{2 \pi c}{\lambda_{0}} \int_{z=0}^{z=L} d z\left[\frac{1}{v_{p}\left(z, \lambda_{0}\right)}-\frac{1}{v_{g}\left(z, \lambda_{0}\right)}\right] \\
& =2 \pi \int_{z=0}^{z=L} d z \frac{\partial n\left(z, \lambda_{0}\right)}{\partial \lambda_{0}},
\end{aligned}
$$

where $v_{p}\left(z, \lambda_{0}\right)=c / n\left(z, \lambda_{0}\right) \quad$ and $\quad v_{g}\left(z, \lambda_{0}\right)=v_{p}\left(z, \lambda_{0}\right)-$ $\lambda_{0} \partial v_{p}\left(z, \lambda_{0}\right) / \partial \lambda_{0}$ are the phase and group velocities inside the HHG target gas, respectively, with $n\left(z, \lambda_{0}\right)$ being the refractive index. The dependence on the propagation direction stems from the pressure profile inside the target. Note that Eq. (1) is a linear approximation of $\Delta \Phi$, which disregards possible nonlinear contributions such as the intensity dependence of $n\left(z, \lambda_{0}\right)[43]$. The pressure-dependent refractive index consists of a contribution from neutral atoms as well as from free electrons. It is given by [27]

$$
n\left(P, \lambda_{0}\right)=1+P(1-\eta)\left(n_{0}-1\right)-\frac{P \rho_{\mathrm{atm}} \eta r_{0} \lambda_{0}^{2}}{2 \pi}
$$

where $\eta$ is the ion fraction, $n_{0}$ is the refractive index at $\lambda_{0}$ at atmosphere, $\rho_{\text {atm }}$ is the number density at atmosphere, and $r_{0}$ is the classical electron radius.

Our goal is to investigate how the CEP of a longwavelength, few-cycle driver pulse is influenced by the high target-gas pressures required for phase matching of waterwindow harmonics. To do so, we make use of the strong CEP dependence of half-cycle cutoff features.

\section{RESULTS}

Our experimental setup is described in detail in Ref. [29]. In brief, 40-fs pulses at $800 \mathrm{~nm}$ from an amplified Ti:sapphire system running at $1 \mathrm{kHz}$ repetition rate are frequency converted in an optical parametric amplifier (HE-TOPAS, Light Conversion). The 40 -fs idler at $1.85 \mu \mathrm{m}$ with a pulse energy of $1 \mathrm{~mJ}$ is focused into a hollow-core fiber for spectral broadening. Pulse compression to $12 \mathrm{fs}$ is achieved with linear propagation through $3 \mathrm{~mm}$ of glass [44], resulting in two-cycle pulses with an energy of up to $350 \mu \mathrm{J}$ on target. The CEP was monitored in an $f-2 f$ setup and actively controlled with a slow feedback loop, resulting in an rms CEP jitter of $200 \mathrm{mrad}$ throughout the measurement.

For HHG, the few-cycle pulses were focused into a closedoff tube target (outer diameter $1.5 \mathrm{~mm}$, inner diameter $0.5 \mathrm{~mm}$, $\sim 300 \mu \mathrm{m}$ entrance and exit holes) using an $f=100 \mathrm{~mm}$ spherical mirror. A 200-nm-thick titanium filter was used to block the fundamental radiation. Water window highorder harmonics were detected in a home-built spectrograph consisting of a controllable slit, an imaging reflective gold grating with 2400 lines / mm (Hitachi), and a cooled CCD x-ray camera (Princeton Instruments).

Figures 1(a) and 1(b) show HCO traces measured at backing pressures of 3.0 and 5.0 bars, respectively. We carefully scanned the CEP in steps of $0.02 \pi \mathrm{rad}$ and recorded highorder-harmonic spectra at each CEP with an integration time of less than $5 \mathrm{~s}$. The expected $\pi$ periodicity of the high-orderharmonic spectra can be clearly seen. The decreasing signal at around $285 \mathrm{eV}$ originates from absorption at the carbon edge from hydrocarbon contamination in the vacuum chamber.

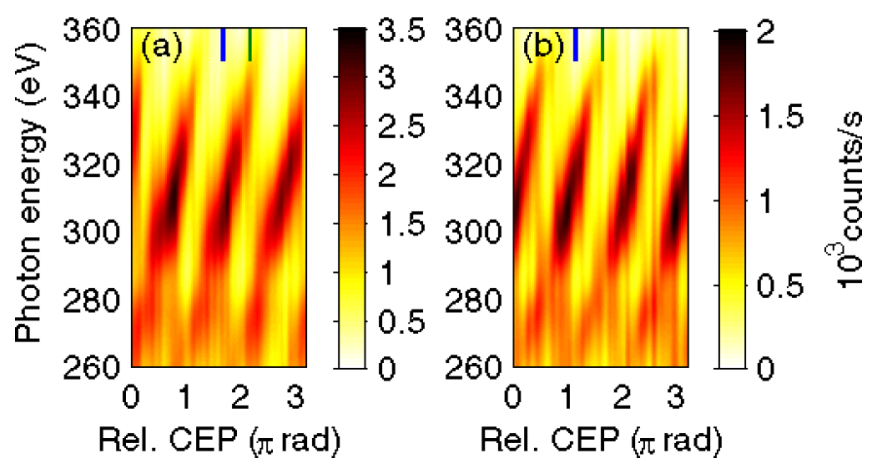

FIG. 1. (Color online) Half-cycle cutoff traces generated with backing pressures of (a) 3.0 bars and (b) 5.0 bars of neon. The relative CEP was scanned with a step size of $0.02 \pi \mathrm{rad}$, and integration times were (a) $4.0 \mathrm{~s}$ and (b) $3.0 \mathrm{~s}$. A 200-nm-thick Ti filter was used to block the fundamental radiation. Color scales are given in $10^{3}$ counts $/ \mathrm{s}$ at the detector. 


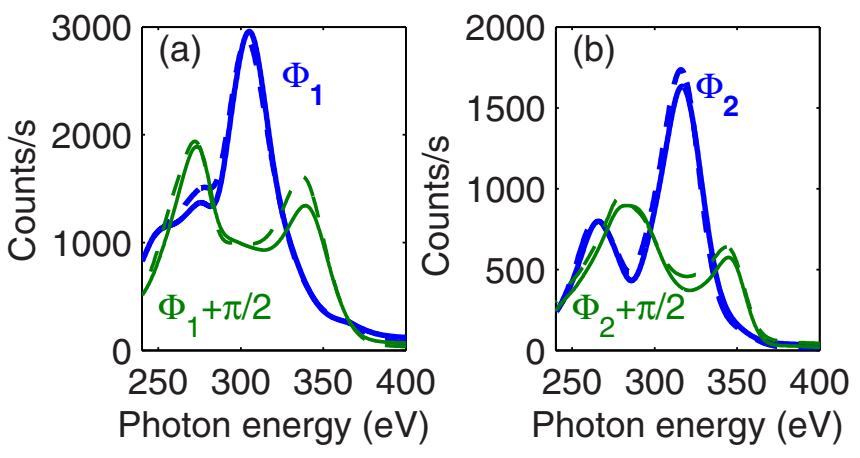

FIG. 2. (Color online) Photon spectra (given in counts/s at the detector) from the HCO traces in Fig. 1 at four different CEP values [at (a) 3.0 bars and (b) 5.0 bars]. The solid spectra correspond to the indicated CEP values in Fig. 1 and are $\pi$ rad apart. The dashed spectra are each $\pi / 2$ rad apart from the solid spectra of the same line width.

At the higher backing pressure of 5.0 bars [Fig. 1(b)], the main features of the $\mathrm{HCO}$ trace remain unchanged. The slightly lower count rate is mostly attributed to reabsorption of the harmonics by residual neon gas in the generation chamber [29].

The excellent spectral control provided by the CEP-stable, few-cycle driving pulses is highlighted in Fig. 2. The solid thick blue and thin green curves represent lineouts of the HCO traces at the CEP values indicated in Fig. 1. They are about $\pi / 2 \mathrm{rad}$ apart and demonstrate that by carefully changing the CEP, spectral tailoring in the water window becomes feasible. This is important in spectroscopic measurements in which spectral stability affects the signal-to-noise ratio, especially close to absorption edges. In addition, the dashed lines in Fig. 2 represent lineouts at CEP values that are $\pi \mathrm{rad}$ apart from their solid-line counterparts, which emphasizes the reproducibility of the high-order-harmonic spectra during the $\mathrm{HCO}$ scans, regardless of the backing pressure.

The HCO traces shown in Fig. 1 are from a set of traces we acquired at backing pressures ranging from 2.0 to 5.0 bars, in steps of 0.5 bar. Each scan was started at the same CEP reference value $\Phi_{0}$ (in Fig. 1 we assigned the value $\Phi_{0}=0$ arbitrarily to $\Phi_{0}$ ). This means that each scan covered the same range of CEP values of the pulses entering the HHG target. With this in mind, it is interesting to note that despite covering the same absolute CEP values, the patterns of HCO traces at different backing pressures are offset from one another with respect to CEP. To highlight this, we plot in Fig. 3(a) the normalized spectra for a CEP of $\Phi_{0}$ with backing pressures of 3.0 bars (thick blue curve) and 5.0 bars (thin green curve). Clearly, the spectral shapes differ strongly from one another. In contrast, if we choose the spectrum generated with a CEP of $\Phi_{0}+0.4 \pi \mathrm{rad}$ with a backing pressure of 5.0 bars, the spectral shapes are remarkably similar, as shown in Fig. 4(b). This implies that despite an equivalent absolute CEP of the pulses entering the HHG target, the in situ CEP values that determine the $\mathrm{HCO}$ traces vary with backing pressure.

To investigate this more quantitatively, we follow a procedure indicated in Figs. 4(a) and 4(b). For each CEP value in the $\mathrm{HCO}$ traces we integrate the harmonic signal between 300 and $320 \mathrm{eV}$. The solid thick blue curve in Fig. 4(a) traces the resulting signal modulation for a backing pressure of 3.0 bars,

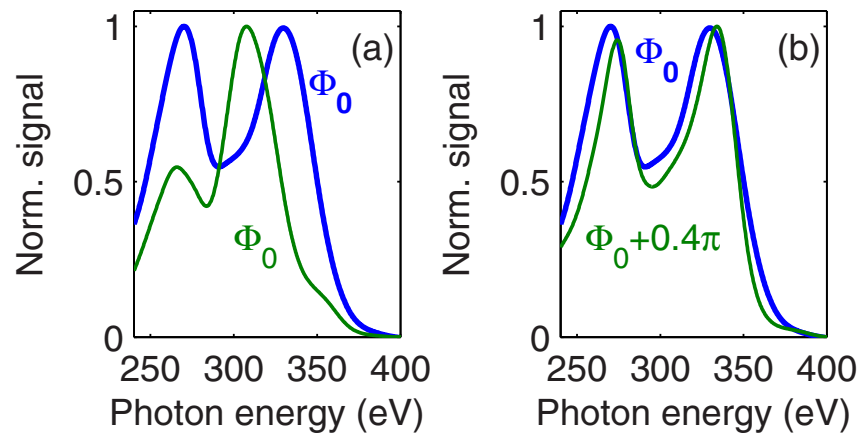

FIG. 3. (Color online) Normalized photon spectra with backing pressures of 3.0 bars (thick blue curve) and 5.0 bars (thin green curve). (a) Spectra with a CEP corresponding to $0 \pi \mathrm{rad}$ in Fig. 1, i.e., with a CEP of $\Phi_{0}$, with clear spectral differences. (b) Spectra with CEPs of $\Phi_{0}$ (3.0 bars, thick blue curve) and $\Phi_{0}+0.4 \pi$ (5.0 bars, thin green curve), which exhibit strong spectral similarity.

and in Fig. 4(b) this is shown for a backing pressure of 5.0 bars (solid thin green curve). The overlaid dashed curves are cosine fits with $\pi$ periodicity to the modulated signal using the function $\cos \left(2 \Phi_{\text {rel }}+\Phi_{\text {off }}\right)$. Here, $\Phi_{\text {rel }}$ are the relative CEP values from the measurement in Fig. 1, and $\Phi_{\text {off }}$ determines the modulation offset of the fit. As indicated by the vertical dashed lines, the fits allow us to extract a relative offset difference $\Delta \Phi_{\text {off }}$ between the signal modulations at different backing pressures. Note that due to the $\pi$ periodicity of the signal the indicated offset difference in Figs. 4(a) and 4(b) is half the value of the corresponding fits.
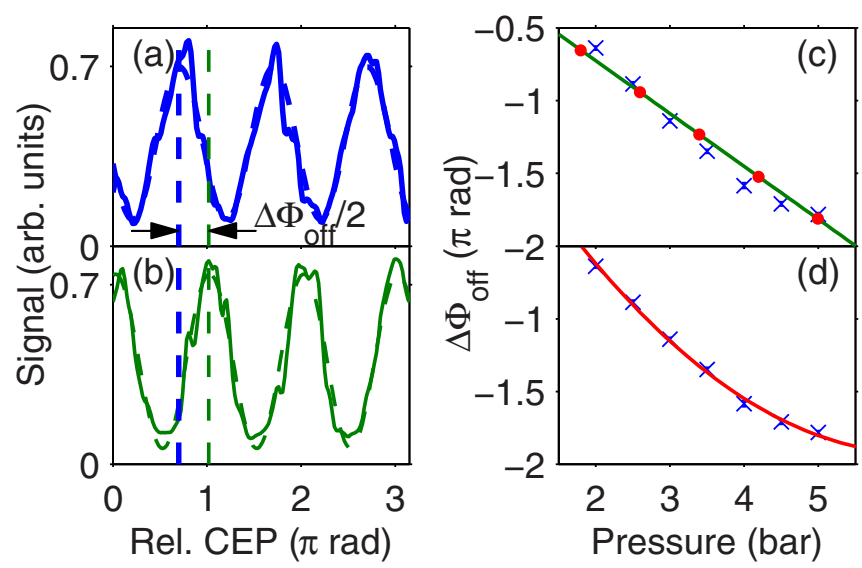

FIG. 4. (Color online) High-order-harmonic signal from the $\mathrm{HCO}$ traces in Fig. 1 integrated from 300 to $320 \mathrm{eV}$ as a function of relative CEP for a backing pressure of (a) 3.0 bars and (b) 5.0 bars. The dashed curves are cosine fits which allow us to determine a relative modulation offset $\Delta \Phi_{\text {off }}$ as shown by the vertical dashed lines. (c) The modulation offsets for measurements with backing pressures between 2.0 and 5.0 bars are given by the crosses. The solid green line represents the calculated modulation offset according to Eq. (1) using Eq. (2) for the pressure-dependent refractive index. The red dots indicate the same calculation, except considering the effect of only the free electrons. The fit parameter for the slope is an average ion fraction (see main text). The vertical position of the experimental data points is adjusted to match the calculated curve. (d) The solid red curve is a second-order polynomial fit to the same data as in (c). 
The relative CEP offsets of all measurements in the backingpressure range between 2.0 and 5.0 bars are plotted as blue crosses in Fig. 4(c). They follow a roughly linear trend which we now wish to analyze based on the consideration presented in Sec. II.

We use Eq. (1) to calculate the CEP phase slip at a given target pressure, using Eq. (2) to determine the phase and group velocities. Across the inner diameter of the target, i.e., between $z=0.5 \mathrm{~mm}$ and $z=1.0 \mathrm{~mm}$, we approximate the target pressure with the backing pressure. Outside the target, i.e., at $z=0 \mathrm{~mm}$ and at $z=1.5 \mathrm{~mm}$, we assume vacuum and interpolate the pressure linearly in between. With this simple model the linear green curve in Fig. 4(c) is obtained. The rather satisfying agreement is achieved with an average ion fraction of $\eta=0.0045$ in Eq. (2), which does not take into account the nonconstant intensity across the pressure profile. However, it results in a very good match of the model with experimental data. Note that since the measurement yields only a relative CEP slip as a function of pressure, the vertical position of the experimental data points in Fig. 4(c) is adjusted to match the calculated curve.

Using Eq. (4) of Ref. [45] to calculate the ionization rate $w(t), \eta=0.0045$ corresponds to a peak intensity of $3.9 \times 10^{14}$ $\mathrm{W} / \mathrm{cm}^{2}$. This is in very good agreement with an experimentally derived peak intensity of about $3.6 \times 10^{14} \mathrm{~W} / \mathrm{cm}^{2}$ based on the highest detected photon energy of $385 \mathrm{eV}$ in Fig. 3.

Interestingly, the main contribution to $\Delta \Phi_{\text {off }}$ in this model comes from the free electrons. This becomes apparent when setting the contribution of the neutral atoms in Eq. (2) to zero, which yields a very similar result, as indicated by the red dots in Fig. 4(c).

The linear model of Fig. 4(c) is a lowest- order approximation to the CEP slip mediated by the free electrons. On closer inspection of Fig. 4(c), however, we find a slight deviation from the lowest-order fit. Hence, we fit a second-order polynomial to our data, shown by the solid red curve in Fig. 4(d). Since we established that the phase slip is dominated by the free electrons, we consider only their contribution here, similar to the dots in Fig. 4(c). Based on a linearly increasing target pressure, the only other parameter from Eq. (2) that may change with the target pressure to result in a nonlinear trend of the phase slip is the ion fraction. In fact, according to the nonlinear fit we can determine that in this model $\eta$ decreases from $\eta=0.0075$ at 2.0 bars to $\eta=0.0023$ at 5.0 bars. Qualitatively, we relate this decreasing ion fraction to plasma defocusing [46] as follows.

With respect to the fit in Fig. 4(d), a decreasing ion fraction as a function of pressure indicates a lower intensity of the driving pulse at higher pressures compared with lower pressures. Since plasma defocusing has precisely this effect, we relate the nonlinear curvature in Fig. 4(d) to plasma defocusing. This analysis is supported by a comparison of the length of the constant-pressure region of our target (inner diameter of $0.5 \mathrm{~mm}$ ) with the defocusing length $L_{D F}$ [46]. At a backing pressure of 2.0 bars we calculate $L_{D F}=0.88 \mathrm{~mm}$, whereas at 5.0 bars we find $L_{D F}=0.35 \mathrm{~mm}$, suggesting that in the high-pressure range of our data the defocusing length is, in fact, shorter than the inner diameter of our target and thus becomes a decisive quantity [46]. Using the nonlinear model, the average ion fraction of $\eta=0.0045$ from Fig. 4(c) corresponds to a backing pressure of 3.7 bars and a defocusing length of $L_{D F}=0.47 \mathrm{~mm}$. The linear model in Fig. 4(c) is thus based on an ion fraction for which the defocusing length is very similar to the inner diameter of the target. However, since at higher pressures defocusing becomes more significant, the linear model is insufficient to capture the nonlinear trend in this pressure region.

Note that the described straightforward procedure permits direct identification of the in situ CEP slip during HHG. In addition, it allows for the extraction of the integrated ionization fraction as well as the peak laser intensity on target. We are able to obtain this information for HHG in the water-window soft $\mathrm{X}$-ray regime by taking advantage of the strong wavelength dependence of the free-electron contribution to the refractive index of the medium.

\section{DISCUSSION}

We now turn to a discussion of the obtained results, restricting our further analysis to the linear model in Fig. 4(c). The interesting conclusion we draw from Fig. 4(c) is that for long wavelengths it is the free electrons that cause an in situ CEP slip in a high-pressure HHG target. As elaborated in Sec. II, free electrons are generated during tunnel ionization of HHG. This implies that our findings reveal an important intensity-to-phase coupling mechanism in HHG which is prominent with long-wavelength, few-cycle pulses. It has direct implications for attosecond-pulse generation in the soft $\mathrm{x}$-ray regime.

To analyze this quantitatively, we consider in the following a target environment of constant pressure. In this case, the dispersion-induced in situ CEP slip is linear in both propagation distance and target pressure according to Eqs. (1) and (2). We can therefore calculate the induced CEP slip per unit length propagation and per unit pressure, i.e., $\Delta \Phi /($ bar $\mathrm{mm})$, and differentiate it with respect to the peak intensity to determine the effect of intensity fluctuations. In order to illustrate the effect in a more general context, the calculation is performed for a range of peak intensities and driving wavelengths. In terms of intensities, we only consider those values that yield a final ionization fraction of 0.005 or less to account for depletion, a reasonable value with respect to typical critical ionization fractions [27]. Moreover, the pulse duration is kept constant at two cycles (FWHM) for all wavelengths.

An intensity variation results in a varying CEP slip through the intensity dependence of the ionization fraction. In our model, we determine the magnitude by multiplying $\partial \Delta \Phi / \partial I$ by a realistic fraction of 0.01 of a given peak intensity value, thus assuming a $1 \%$ intensity fluctuation. Figure 5(a) displays the result of the dispersion-induced CEP slip for neon, as in our experiment, for selected wavelengths of $0.8 \mu \mathrm{m}$ (solid thick blue curve), $1.85 \mu \mathrm{m}$ (solid thin red curve), $2.5 \mu \mathrm{m}$ (dashed green curve), and $3.5 \mu \mathrm{m}$ (dash-dotted orange curve).

We find that under the mentioned conditions an intensityinduced CEP slip is not significant in the short-wavelength limit of $0.8 \mu \mathrm{m}$ (solid thick blue curve). Due to the strong wavelength dependence of the dispersion in Eq. (2), however, the effect becomes prominent even at moderate peak intensities of around $3 \times 10^{14} \mathrm{~W} / \mathrm{cm}^{2}$ for wavelengths of $2.5 \mu \mathrm{m}$ and 


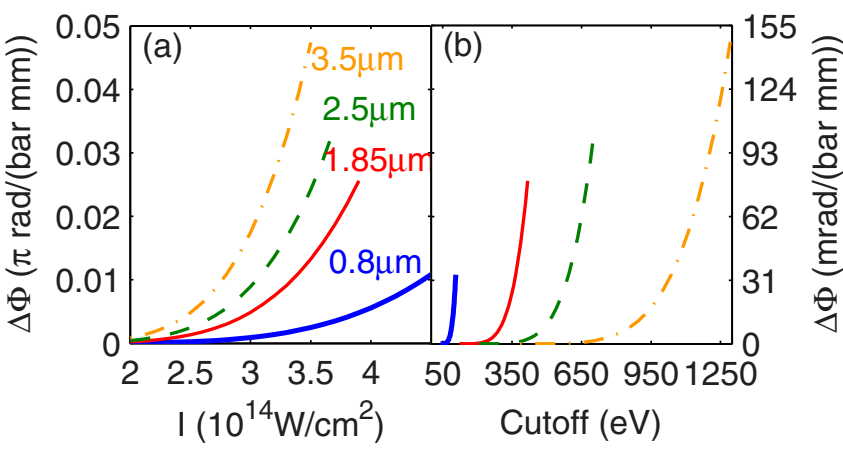

FIG. 5. (Color online) (a) Calculated dispersion-induced CEP slip in neon as a function of peak intensity and (b) corresponding single-atom cutoff, assuming a peak intensity fluctuation of $1 \%$. The CEP slip is calculated per bar of gas pressure (constant pressure profile) and per millimeter of propagation. The axis on the left indicates the CEP slip in units of $\pi \mathrm{rad} /($ bar $\mathrm{mm})$, and the right axis is in units of $\mathrm{mrad} /($ bar $\mathrm{mm}$ ) for both plots. Plots show the results for selected wavelengths of $0.8 \mu \mathrm{m}$ (solid thick blue curve), $1.85 \mu \mathrm{m}$ (solid thin red curve), $2.5 \mu \mathrm{m}$ (dashed green curve), and $3.5 \mu \mathrm{m}$ (dash-dotted orange curve). Only peak intensities are considered that yield a final ionization fraction of 0.005 or less.

above. For example, at $2.5 \mu \mathrm{m}$ and a peak intensity of $3.5 \times 10^{14} \mathrm{~W} / \mathrm{cm}^{2}$ CEP fluctuations of $76 \mathrm{mrad} /($ bar $\mathrm{mm})$ $[\sim 0.024 \pi \mathrm{rad} /($ bar $\mathrm{mm})]$ can be expected from the assumed intensity jitter of $1 \%$. Keeping in mind that in neon multiatmosphere target pressures are typically required for phase matching, this value can correspond to a significant uncertainty in the absolute CEP value of a few-cycle pulse with direct consequences for the generation of attosecond pulses. As shown in Fig. 2, large CEP changes strongly influence the generated high-order-harmonic spectrum and therefore also the temporal profile of the emitted attosecond pulses. For applications that rely on a certain attosecond-pulse duration at a given photon energy, such fluctuations clearly have detrimental effects. Furthermore, a CEP jitter can result in the degradation of pump-probe measurements on the attosecond time scale. If the investigated dynamics are triggered by the electric field of a femtosecond pump pulse that is split off from the HHG driving pulse prior to HHG, the intensity-caused CEP slip does not affect the pump arm of the experiment, only the HHG process. In this case, a CEP jitter directly translates into a temporal uncertainty of the pump-probe measurement, amounting to $\sim 100 \mathrm{as} /($ bar $\mathrm{mm}$ ) in the example above (HHG at $2.5 \mu \mathrm{m}$, peak intensity of $3.5 \times 10^{14} \mathrm{~W} / \mathrm{cm}^{2}$ ).

In Fig. 4(b) the same calculations are expressed as a function of the single-atom cutoff [32,33] corresponding to the peak intensities shown in Fig. 4(a). We note that if a certain cutoff can be used with different combinations of intensity and square of the wavelength, i.e., identical $U_{p}$, a larger driver wavelength results in a smaller intensity-tophase coupling. This dependence arrives from the quadratic wavelength dependence of the cutoff, allowing for a reduced peak intensity and thus a lower free-electron density at longer wavelengths. We note that the presented calculations yield similar results for argon (helium), albeit at lower (higher) peak intensities. For all three gases (Ar, He, Ne) we find that at experimentally viable peak intensities a realistic intensity jitter of $1 \%$ can result in a dispersion-induced CEP fluctuation with substantial influence on the generation of attosecond pulses and their application in pump-probe experiments. The effect should be taken into account when working with few-cycle pulses at long wavelength, in terms of both the laser system parameters and the HHG target design.

\section{CONCLUSION}

We demonstrated the importance of intensity-to-phase coupling for ponderomotively scaled $\mathrm{HHG}$ in the soft x-ray regime and discussed the effects on reproducible singleattosecond-pulse generation in the water window. We presented high-order-harmonic spectra generated with two-cycle, CEP-controlled pulses at $1.85 \mu \mathrm{m}$ in a high-pressure neon target, allowing us to detect half-cycle cutoff features at photon energies of up to $385 \mathrm{eV}$. HCO traces acquired in the backingpressure range between 2.0 and 5.0 bars exhibit a relative shift of the covered CEP range. Using a simple model based on the pressure-dependent dispersion of the target gas, we could establish that the observed effect on an in situ CEP slip of the driving pulse is predominantly mediated by the generated free electrons. Beyond a linear approximation of our model, a nonlinear trend in the observed data could be related to plasma defocusing of the driving pulse at high target pressures. In a generalized analysis we elaborated that due to the strong intensity dependence of the tunnel ionization process intensity fluctuations of only $1 \%$ can turn the dispersion-induced CEP slip into a significant CEP jitter with deteriorating effects for the generation of attosecond pulses and their applications in pump-probe experiments. Future HHG experiments with few-cycle, long-wavelength pulses should take this finding into account. Our investigation highlights the importance of CEP slips for the next generation of attosecond sources which operate in a high-pressure regime.

\section{ACKNOWLEDGMENTS}

This work was supported by the Ministerio de Economía y Competitividad through Plan Nacional (FIS2011-30465-C0201), the Catalan Agencia de Gestio d'Ajuts Universitaris i de Recerca (AGAUR) with SGR 2014-2016, Fundacio Cellex Barcelona, assistance from Fundação para a Ciência e a Tecnologia (Grant No. SFRH/BD/69913/2010), and funding from LASERLAB-EUROPE, Grant Agreement 284464.
[1] P. B. Corkum and F. Krausz, Nat. Phys. 3, 381 (2007).

[2] F. Krausz and M. Y. Ivanov, Rev. Mod. Phys. 81, 163 (2009).

[3] R. L. Sandberg, A. J. Paul, D. Raymondson, S. Hädrich, D. Gaudiosi, J. Holtsnider, R. Tobey, O. Cohen, M. M. Murnane,
H. C. Kapteyn, C. Song, J. Miao, Y. Liu, and F. Salmassi, Phys. Rev. Lett. 99, 098103 (2007).

[4] P. M. Paul, E. S. Toma, P. Breger, G. Mullot, F. Auge, P. Balcou, H. G. Muller, and P. Agostini, Science 292, 1689 (2001). 
[5] K. Klünder, J. Dahlström, M. Gisselbrecht, T. Fordell, M. Swoboda, D. Guénot, P. Johnsson, J. Caillat, J. Mauritsson, A. Maquet, R. Taïeb, and A. L'Huillier, Phys. Rev. Lett. 106, 143002 (2011).

[6] M. Hentschel, R. Kienberger, Ch. Spielmann, G. A. Reider, N. Milosevic, T. Brabec, P. B. Corkum, U. Heinzmann, M. Drescher, and F. Krausz, Nature (London) 414, 509 (2001).

[7] F. Ferrari, F. Calegari, M. Lucchini, C. Vozzi, S. Stagira, G. Sansone, and M. Nisoli, Nat. Photonics 4, 875 (2010).

[8] K. Zhao, Q. Zhang, M. Chini, Y. Wu, X. Wang, and Z. Chang, Opt. Lett. 37, 3891 (2012).

[9] J. A. Wheeler, A. Borot, S. Monchocé, H. Vincenti, A. Ricci, A. Malvache, R. Lopez-Martens, and F. Quéré, Nat. Photonics 6, 829 (2012).

[10] F. Silva, S. M. Teichmann, S. L. Cousin, M. Hemmer, and J. Biegert, Nat. Commun. 6, 6611 (2015).

[11] M. Drescher, M. Hentschel, R. Kienberger, M. Uiberacker, V. S. Yakovlev, A. Scrinzi, T. Westerwalbesloh, U. Kleineberg, U. Heinzmann, and F. Krausz, Nature (London) 419, 803 (2002).

[12] M. Uiberacker, T. Uphues, M. Schultze, A. J. Verhoef, V. S. Yakovlev, M. F. Kling, J. Rauschenberger, N. M. Kabachnik, H. Schröder, M. Lezius, K. L. Kompa, H. G. Muller, M. J. J. Vrakking, S. Hendel, U. Kleineberg, U. Heinzmann, M. Drescher, and F. Krausz, Nature (London) 446, 627 (2007).

[13] M. Schultze et al., Science 328, 1658 (2010).

[14] F. Lépine, M. Y. Ivanov, and M. J. J. Vrakking, Nat. Photonics 8, 195 (2014).

[15] G. Sansone, F. Kelkensberg, J. F. Pérez-Torres, F. Morales, M. F. Kling, W. Siu, O. Ghafur, P. Johnsson, M. Swoboda, E. Benedetti, F. Ferrari, F. Lépine, J. L. Sanz-Vicario, S. Zherebtsov, I. Znakovskaya, A. L'Huillier, M. Y. Ivanov, M. Nisoli, F. Martín, and M. J. J. Vrakking, Nature (London) 465, 763 (2010).

[16] F. Calegari, D. Ayuso, A. Trabattoni, L. Belshaw, S. De Camillis, S. Anumula, F. Frassetto, L. Poletto, A. Palacios, P. Decleva, J. B. Greenwood, F. Martin, and M. Nisoli, Science 346, 336 (2014).

[17] A. L. Cavalieri, N. Müller, T. Uphues, V. S. Yakovlev, A. Baltuška, B. Horvath, B. E. Schmidt, L. Blümel, R. Holzwarth, S. Hendel, M. Drescher, U. Kleineberg, P. M. Echenique, R. Kienberger, F. Krausz, and U. Heinzmann, Nature (London) 449, 1029 (2007).

[18] M. Schultze, E. M. Bothschafter, A. Sommer, S. Holzner, W. Schweinberger, M. Fiess, M. Hofstetter, R. Kienberger, V. Apalkov, V. S. Yakovlev, M. I. Stockman, and F. Krausz, Nature (London) 493, 75 (2013).

[19] M. Schultze, K. Ramasesha, C. D. Pemmaraju, S. A. Sato, D. Whitmore, A. Gandman, J. S. Prell, L. J. Borja, D. Prendergast, K. Yabana, D. M. Neumark, and S. R. Leone, Science 346, 1348 (2014).

[20] D. S. Steingrube, E. Schulz, T. Binhammer, M. B. Gaarde, A. Couairon, U. Morgner, and M. Kovačev, New J. Phys. 13, 043022 (2011).

[21] V. S. Yakovlev, M. Y. Ivanov, and F. Krausz, Opt. Express 15, 15351 (2007).

[22] M. V. Frolov, N. L. Manakov, and A. R. Starace, Phys. Rev. Lett. 100, 173001 (2008).
[23] E. L. Falcão Filho, M. Gkortsas, A. Gordon, and F. X. Kärtner, Opt. Express 17, 11217 (2009).

[24] D. R. Austin and J. Biegert, Phys. Rev. A 86, 023813 (2012).

[25] B. Shan and Z. Chang, Phys. Rev. A 65, 011804 (2001).

[26] J. Tate, T. Auguste, H. G. Muller, P. Salières, P. Agostini, and L. F. DiMauro, Phys. Rev. Lett. 98, 013901 (2007).

[27] T. Popmintchev, M.-C. Chen, A. Bahabad, M. Gerrity, P. Sidorenko, O. Cohen, I. P. Christov, M. M. Murnane, and H. C. Kapteyn, Proc. Natl. Acad. Sci. USA 106, 10516 (2009).

[28] M.-C. Chen, P. Arpin, T. Popmintchev, M. Gerrity, B. Zhang, M. Seaberg, D. Popmintchev, M. M. Murnane, and H. C. Kapteyn, Phys. Rev. Lett. 105, 173901 (2010).

[29] S. L. Cousin, F. Silva, S. M. Teichmann, M. Hemmer, B. Buades, and J. Biegert, Opt. Lett. 39, 5383 (2014).

[30] J.-F. Adam, J.-P. Moy, and J. Susini, Rev. Sci. Instrum. 76, 091301 (2005).

[31] T. Popmintchev, M.-C. Chen, D. Popmintchev, P. Arpin, S. Brown, S. Alisauskas, G. Andriukaitis, T. Balciunas, O. D. Mücke, A. Pugzlys, A. Baltuška, B. Shim, S. E. Schrauth, A. Gaeta, C. Hernández-García, L. Plaja, A. Becker, A. JaronBecker, M. M. Murnane, and H. C. Kapteyn, Science 336, 1287 (2012).

[32] K. J. Schafer, B. Yang, L. F. DiMauro, and K. C. Kulander, Phys. Rev. Lett. 70, 1599 (1993).

[33] P. B. Corkum, Phys. Rev. Lett. 71, 1994 (1993).

[34] A. Gordon and F. X. Kärtner, Opt. Express 13, 2941 (2005).

[35] R. Kienberger, E. Goulielmakis, M. Uiberacker, A. Baltuška, V. S. Yakovlev, F. Bammer, A. Scrinzi, T. Westerwalbesloh, U. Kleineberg, U. Heinzmann, M. Drescher, and F. Krausz, Nature (London) 427, 817 (2004).

[36] E. Goulielmakis, M. Schultze, M. Hofstetter, V. S. Yakovlev, J. Gagnon, M. Uiberacker, A. L. Aquila, E. M. Gullikson, D. T. Attwood, R. Kienberger, F. Krausz, and U. Kleineberg, Science 320, 1614 (2008).

[37] I. J. Sola, E. Mével, L. Elouga, E. Constant, V. Strelkov, L. Poletto, P. Villoresi, E. Benedetti, J.-P. Caumes, S. Stagira, C. Vozzi, G. Sansone, and M. Nisoli, Nat. Phys. 2, 319 (2006).

[38] C. A. Haworth, L. E. Chipperfield, J. S. Robinson, P. L. Knight, J. P. Marangos, and J. W. G. Tisch, Nat. Phys. 3, 52 (2006).

[39] A. Jullien, T. Pfeifer, M. J. Abel, P. M. Nagel, M. J. Bell, D. M. Neumark, and S. R. Leone, Appl. Phys. B 93, 433 (2008).

[40] L. E. Chipperfield, J. Robinson, P. Knight, J. Marangos, and J. W. G. Tisch, Laser Photonics Rev. 4, 697 (2010).

[41] N. Ishii, K. Kaneshima, K. Kitano, T. Kanai, S. Watanabe, and J. Itatani, Nat. Commun. 5, 3331 (2014).

[42] H. Telle, G. Steinmeyer, A. Dunlop, J. Stenger, D. Sutter, and U. Keller, Appl. Phys. B 69, 327 (1999).

[43] M. J. Ablowitz, B. Ilan, and S. T. Cundiff, Opt. Lett. 29, 1808 (2004).

[44] B. E. Schmidt, P. Bejot, M. Giguere, A. D. Shiner, C. TralleroHerrero, E. Bisson, J. Kasparian, J.-P. Wolf, D. M. Villeneuve, J.-C. Kieffer, P. B. Corkum, and F. Légaré, Appl. Phys. Lett. 96, 121109 (2010).

[45] A. M. Perelomov, V. S. Popov, and M. V. Terentev, Sov. Phys. JETP 23, 924 (1966).

[46] A. Couairon and A. Mysyrowicz, Phys. Rep. 441, 47 (2007). 